\title{
Small-grained detector of ionizing radiation based on $\mathrm{ZnSe}(\mathrm{Te})$
}

\author{
V.G.Volkov, V.P.Gavrilyuk, L.P.Gal'chinetskii, B.V.Grinyov, K.A.Katrunov, V.D.Ryzhikov. \\ Scientific and Technological Centre for Radiation Instrument Concern "Institute for Single Crystals", 60 Lenin ave., 310001 \\ Kharkov, Ukraine
}

\begin{abstract}
A highly efficient $\mathrm{ZnSe}(\mathrm{Te})$ scintillation detector combined with Si-photodiode has been developed. A conglomerate made up of $\mathrm{ZnSe}(\mathrm{Te})$ grains is used as a scintillator. Optimal shape of the grains, reflecting cover and disperse environment type are selected to improve light collection within the detector using numerical simulation. Various shapes of light guides have been considered to maximize light output of the detector.
\end{abstract}

Keywords: small-grained scintillator, $\gamma$-, $\beta$-radiation, light transducer.

Paper received 12.10.99; revised manuscript received 14.01.99; accepted for publication 21.03.00.

\section{Introduction}

Scintillators based on $\mathrm{ZnSe}(\mathrm{Te})$ in combination with silicon photodiode are distinguished by their high scintillation efficiency $\eta(18 \%)$ [1] and good spectral matching coefficient $F=91 \%$. This makes them very promising for detection of beta- and low energy gamma-radiation. Single crystalline plates $\mathrm{ZnSe}(\mathrm{Te})$ in combination with photomultiplier PMT-110 have energy resolution of $\cong 9 \%$ for conversion electrons ${ }^{137} \mathrm{Cs}$ [2]. In addition, crystals $\mathrm{ZnSe}(\mathrm{Te})$ are non-hygroscopic and have high radiation stability (up to $10^{8} \mathrm{rad}$ ). Electron back scattering coefficient $k$ in $\mathrm{ZnSe}(\mathrm{Te})$ is $40-50 \%$.

Present-day preparation techniques of scintillation crystals $\mathrm{ZnSe}(\mathrm{Te})$ do not allow to obtain plates larger than $4 \mathrm{~cm}$ in diameter. An improvement of detector sensitivity can be achieved not only by increasing its area, but also by creation of optimum conditions of light collection in the scintillator.

One of the possible ways to increase the detector area is to make a detector in the form of a conglomerate of small grains of $\mathrm{ZnSe}(\mathrm{Te})$ combined into a single "smallgrained" ("small-crystalline") scintillator. The area of such scintillator is practically unlimited from the point of view of its preparation technology. In this case the problem of optimum light collection conditions becomes vital. Ways of its solution involve optimization of the grain size, the use of additional reflective coatings and transparent disperse medium in which the grains are installed.
In this paper we report a small-grained scintillator based on $\mathrm{ZnSe}(\mathrm{Te})$. Its thickness is $0.15-0.2 \mathrm{~cm}$, which corresponds to $90 \%$ absorption of $50 \mathrm{keV} \gamma$-ray radiation or $2.4 \mathrm{MeV}$ beta-radiation [3]. The grain size was chosen to correspond to the scintillator thickness (singlelayer packing). Multilayer structures with particle size smaller than the scintillator thickness have low output efficiency of intrinsic radiation due to light losses on the grain boundaries.

\section{Calculation of optimum light collection conditions in a $\mathrm{ZnSe}$ grain}

Using the Monte-Carlo (MC) method, calculations of optimum light collection conditions in the small-grained $\mathrm{ZnSe}(\mathrm{Te})$ scintillator were carried out. The MC procedures for calculation of light collection in scintillation detectors have been described in detail [4]. For the calculations, we assumed that grain sides were mirror-smooth (cleavage surfaces). Two limiting cases were considered of the location of scintillation flashed inside the grain: a) high penetrating ability of the detected radiation - the flashes are uniformly distributed inside the crystal; $b$ ) low penetrating ability of the detected radiation, which is completely absorbed by the thin layer adjacent to the crystal surface (the layer thickness is small as compared with characteristic dimensions of the crystal). For intermediate values of penetrating ability, the light collection coefficient $\tau$ is between these limiting cases. 


\section{V.G. Volkov et al.: Small-grained detector of ionizing radiation based on $\mathrm{ZnSe}(\mathrm{Te})$}

Several shapes of $\mathrm{ZnSe}(\mathrm{Te})$ grains, which are easily obtainable practically, have been considered: parallelogram, parallelogram with rounded upper side, tetrahedral pyramid, trihedral and hexahedral prisms, and a hemisphere. In Table 1 calculated values $\tau$ are presented for grains of these shapes, both without additional reflective coating and for two cases of reflective coatings. These cases were: a) diffusely scattering coating without optical contact with crystal surface and reflection coefficient $R=0.9$, which is realized with Teflon reflectors (reflector 1$)$; b) reflective coating in optical contact with the crystal surface, $R=0.9$ (reflector 2 ), which corresponds to covering of the crystal surface with $\mathrm{MgO}$ powder.

Calculation results accounting for effects of the adhesive composition with $n=1.5$ and absorption coefficient $\alpha=0.05 \mathrm{~cm}^{-1}$ upon light transmission from the output window to the light sensitive surface of the silicon photodiode with $n=4$ showed that $68 \%$ of the total light passed through the output window of the scintillator are reflected from the boundary "crystal-adhesive"; 6.7\% are reflected from the boundary "adhesive - photodiode surface"; $0.3 \%$ are absorbed in the bulk of adhesive, and only $25 \%$ interact with the light-sensitive surface of the photodiode. For adhesives with $n=1.6$ this value increases up to $30 \%$, and with $n=1.8$ - even $40 \%$. Filling with adhesive of the space above the output window leads to a decrease in $\tau$ with higher filling levels.
It can be concluded from these results:

- maximum $\tau$ value for a single crystalline grain of $\mathrm{ZnSe}(\mathrm{Te})$ is reached when it is pyramid-shaped with side inclination angle to the base $60^{\circ}$;

- light collection for all the considered crystal shapes is improved in the presence of light-reflecting coating, degree of this improvement is substantially different with different shapes;

- filling the space between grains with an optical adhesive leads to a decrease in the light output;

- the fraction of intrinsic radiation that interacts with the sensitive surface of the photodiode substantially increases with higher $n$ of the adhesive ensuring the optical contact between the detector output window and the photodiode;

- application of the reflective coating onto the grain surface favors rise in integral light output of the smallgrained scintillator.

\section{Efficiency of a small-grained $\mathrm{ZnSe}(\mathrm{Te})$ scintillator}

Accounting for the results of computer calculations aimed at optimization of light collection conditions 4 samples of small-grained $\mathrm{ZnSe}(\mathrm{Te}$ ) scintillator were prepared, 3.5 $\mathrm{cm}$ in diameter and $0.15 \mathrm{~cm}$ thick:

Table 1. Light collection coefficients of $\mathrm{ZnSe}(\mathrm{Te})$ grains of different shapes and types of reflective coatings

\begin{tabular}{|c|c|c|c|c|c|c|}
\hline Shape of grains & $\begin{array}{l}\text { without } \\
\text { reflector }\end{array}$ & reflector 1 & reflector 2 & $\begin{array}{l}\text { without } \\
\text { reflector }\end{array}$ & reflector 1 & reflector 2 \\
\hline & \multicolumn{3}{|c|}{$\begin{array}{l}\text { the flashes uniformly distributed inside } \\
\text { a grain }\end{array}$} & \multicolumn{3}{|c|}{$\begin{array}{l}\text { the flashes absorbed by the thin layer } \\
\text { adjacent to the grain surface }\end{array}$} \\
\hline $\begin{array}{l}\text { Tetrahedral } \\
\text { pyramid }\end{array}$ & 0,600 & 0,815 & 0,590 & 0,668 & 0,836 & 0,599 \\
\hline Hemisphere & 0,437 & 0,508 & 0,536 & 0,627 & 0,656 & 0,548 \\
\hline $\begin{array}{l}\text { Trihedral prism } \\
(\text { pattern } 1)^{*}\end{array}$ & 0,357 & 0,447 & 0,484 & 0,378 & 0,461 & 0,470 \\
\hline $\begin{array}{l}\text { Trihedral prisms } \\
\text { (pattern } 2)^{*}\end{array}$ & 0,147 & 0,177 & 0,263 & 0,146 & 0,180 & 0,256 \\
\hline $\begin{array}{l}\text { Hexahedral prism } \\
\text { (pattern } 1)^{*}\end{array}$ & 0,202 & 0,258 & 0,190 & 0,221 & 0,264 & 0,193 \\
\hline $\begin{array}{l}\text { Hexahedral prism } \\
(\text { pattern } 2)^{*}\end{array}$ & 0,145 & 0,172 & 0,395 & 0,147 & 0,171 & 0,412 \\
\hline $\begin{array}{l}\text { Parallelogram } \\
\text { with rounded } \\
\text { upper side } * *\end{array}$ & 0,339 & 0,410 & 0,297 & 0,371 & 0,432 & 0,296 \\
\hline Parallelogram & 0,144 & 0,173 & 0,258 & 0,145 & 0,175 & 0,259 \\
\hline
\end{tabular}

*pattern 1 corresponds to the case when one of the sides of the prism is the output window; pattern 2 - when the output window is the prism bottom.

** grain has a shape of parallelogram; the side opposing the output window is a part of cylindrical surface with $r=0.17 \mathrm{~cm}$. 


\section{V.G. Volkov et al.: Small-grained detector of ionizing radiation based on $\mathrm{ZnSe}(\mathrm{Te})$}

sample 1 - formed of several layers of grains of arbitrary size and shape, without reflective coating, in the matrix of an optically transparent polymer medium; it was located inside a metal container with output window of quartz glass;

sample 2 - formed of one layer of grains of the optimized sizes and shapes in the matrix of an optically transparent polymer medium; covered with a special housing, without reflective coating and quartz glass;

sample 3 - the same as the sample 2, but the grains are covered with light-reflective composition;

sample 4 - a single-crystalline $\mathrm{ZnSe}(\mathrm{Te})$ plate.

The efficiency of the detectors was determined by measurement of the output signal by the pulse method in the counting mode. Intrinsic radiation was detected with silicon $p$ - $i$ - $n$ photodiodes S3590 Hamamatsu. ${ }^{137}$ Cs was used as ionizing radiation source. This nuclide, alongside with $662 \mathrm{keV}$ gamma-radiation, does also emit internal conversion electrons. We determined both total number of countings $\left(I_{\text {total }}\right)$ and the number of counts corresponding to gamma-radiation only $\left(I_{\gamma}\right)$. In the latter case, an aluminum disc was installed between the source and detector to cut off beta-radiation. The difference $I_{\beta}=I_{\text {total }}-I_{\gamma}$ characterizes beta-efficiency of the detector, and the value $k=I_{\beta} / I_{\text {total }}$ - its relative sensitivity to betaradiation. X-ray sensitivity of the detector was determined by detection of the radiation with effective energy of quanta $\sim 70 \mathrm{keV}$. As photoreceiver a PD-288 silicon photodiode was used. The light output of the sample 1 was taken to be equal to unity. Measurement results are presented in Table 2. The following conclusions could be made:

- the best sensitivity, both with ${ }^{137} \mathrm{Cs}$ and $70 \mathrm{keV} \mathrm{X}$ ray sources, was observed for the sample 3;

- efficiency of the sample 2 as compared with sample 1 is practically the same for gamma-radiation. At the same time, the sample 2 is much more efficient for detection of beta-radiation;

- the use of reflective coating on grain surfaces (the sample 3) significantly improve the detector efficiency for gamma-radiation, sensitivity to beta-radiation remains practically unchanged;

-efficiency of the single crystalline plate of $\mathrm{ZnSe}(\mathrm{Te})$ is much lower for beta-radiation as compared with the samples 2 and 3. For gamma-radiation, this difference is not so significant.

\section{Calculation of the optimum shape of light beam}

To increase the output signal of the detector, the area of the substrate upon which scintillator grains are placed should be increased. The limit for such an increase is related to a small light sensitive area of Si-photodiodes. Thus, there is a need to use a light transducer to concentrate the scintillation light on the photodetector.

Transmission coefficients were calculated for different types of light transducers using the MC method. The reflection of light from the transducer surface, upon which scintillator grains were located, was considered as reflection from the boundary between media with refraction indexes of 1.5 and 2.57 respectively (light transducer - a $\mathrm{ZnSe}(\mathrm{Te})$ grain). If a light beam enters the grain, probability of its coming back to the transducer was taken as 0.6 (the value of light collection coefficient for the pyramid-shaped grain with the angle between the side and the base $60^{\circ}$ ). The direction of a light beam having entered the transducer was considered as evenly distributed in space (isotropic source).

The transducer efficiency can be characterized by the relationship

$P=\tau S_{2} / S_{1}$

where $S_{2}$ is the area occupied by scintillator grains, $S_{1}$ is the area of the transducer output window connected to the photoreceiver.

It shows how many times the signal from small-grained scintillator with light transducer is stronger than that in the case when scintillator grains are applied directly upon the transducer. The optimum shape for light transducer is wedge-like (Fig.1a).

Scintillator grains are applied onto the surface of the wedge of large area. The back end of the wedge is lightsensitive (coupled with a photodiode). Optimization of the design consists in choosing wedge length $H$ and its width at the far end (from the output window) $l$ to ensure the highest efficiency $P$ as defined by (1). Fig. 2 shows calculated two-dimensional dependence of efficiency $P$ upon the said geometrical factors. It is important that value of $S_{1}$ is practically not limited and should be related only with conditions of specific detector application.

Table 2. Efficiency of $\mathrm{ZnSe}(\mathrm{Te})$ small-grained scintillators for detection of beta-, gamma-radiation of ${ }^{137}$ Cs and X-ray radiation

\begin{tabular}{|c|c|c|c|c|c|}
\hline \multirow[t]{2}{*}{ Sample } & \multicolumn{3}{|c|}{ Number of countings } & \multirow{2}{*}{$\begin{array}{l}\text { X-ray sensitivity, } \\
\text { arb. units }\end{array}$} & \multirow[t]{2}{*}{$\kappa$} \\
\hline & $I_{\text {total }}$ & $\begin{array}{c}I_{\gamma}, \\
E=665 \text { кеВ }\end{array}$ & $\begin{aligned} & I_{b}, \\
E= & 512 \text { кеВ }\end{aligned}$ & & \\
\hline 1 & 1900 & 1700 & 200 & 1,0 & 0,1 \\
\hline 2 & 3500 & 1700 & 1800 & 1,31 & 0,5 \\
\hline 3 & 4600 & 2700 & 1900 & 1,75 & 0,4 \\
\hline 4 & 2700 & 2400 & 300 & - & 0,09 \\
\hline
\end{tabular}




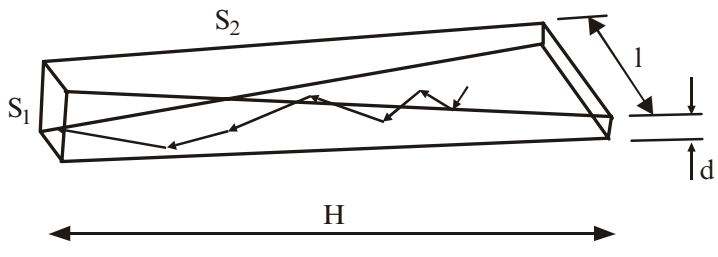

a)

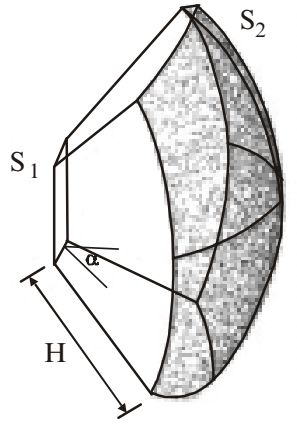

b)

Fig. 1. Concentrating light transducers. $S_{1}-$ area coupled with the photodetector; $S_{2}-$ area covered with scintillator grains; $\mathrm{a}$ - wedge-like transducer (counting mode); $\mathrm{b}$ - transducer with spherical input surface (spectrometry).

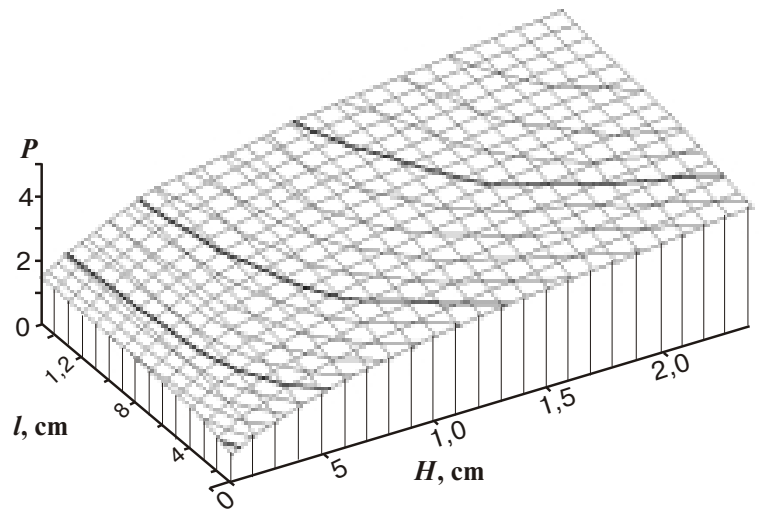

Fig. 2. Efficiency $P$ of the wedge-shaped light transducer as a function of its dimensions - length $H$ and width at the end opposite to the transducer $l$. Dimensions of the output window $S_{1}=$ $1 \times 1 \mathrm{~cm}^{2}$

To use a small-grained detector for spectrometry, the light transducer design should ensure equality of $\tau$ in different points of $S_{1}$. Analysis of different transducer shapes from this point of view showed that the optimum design is concentric in shape with input window in the form of spherical surface element (Fig. 1b). Calculation of $\tau$ for such transducer $(2 \mathrm{~cm}$ length, inclination angle of the sides to the output window $30^{\circ}$ ) results in a contribution of $\sim 2 \%$ of non-uniformity $\tau$ to the energy resolution. Such detectors, as for their total light output $(P=1.17)$, are worse than detectors with wedge-shaped transducers $(P=1.8$ for $S_{2}=10 \mathrm{~cm}^{2}$ ), but are promising for spectrometric applications.

\section{Conclusions}

1. Using light collection model, optimum grain shape was determined for $\mathrm{ZnSe}(\mathrm{Te})$ scintillator, and calcula- tions have been made of the effects of reflective coatings and parameters of the transparent matrix of the smallgrained scintillator upon its sensitivity.

2. Accounting for the results of modelling, smallgrained scintillators were prepared, which were much more sensitive than detectors made of single crystalline plates of the same material.

3. Optimum design of the concentrating light transducer for efficient light collection from the small-grained scintillator of large area onto light sensitive area of the photodiode for operation both in the current mode (wedgeshaped transducer) and in the spectrometric mode (transducer with spherical surface) has been calculated.

4. The results obtained allow to produce highly efficient detectors of beta-, $\mathrm{X}$-ray and soft gamma radiation based on these small-crystalline $\mathrm{ZnSe}(\mathrm{Te})$ scintillators.

\section{Acknowledgements}

The authors are grateful to V.Z.Kvitnitskaya and E.A.Danshin for preparation of the samples and measuring them by the pulse method in the counting mode.

\section{References}

1. K.A. Katrunov, L.P. Galchinetskii, V.D. Ryzhikov et al. Determination of conversion efficiency of X-ray luminophores by measurement of radiation power // Atomnaya Energiya, 76, No.5, p. 428-431 (1994)

2. V.D. Ryzhikov, Yu.A. Borodenko, S.N. Galkin et al. Scintillation crystals $\mathrm{ZnSe}(\mathrm{Te})$ : preparation, propeties, applications. In: Proc. Int. Conference "Inorganic Scintillators and their Applications», p. 465-468, August 28-September 1, 1995, Delft, The Netherlands.

3. V.D. Ryzhikov, V.P. Sokhin, Optimization of scintillator thickness during detection of X-ray and gamma-radiation in a broad energy range // Pribory i Tekhnika Eksp., No.5, p.177181 (1988) (in Russian).

4. V.P. Gavrilyuk, E.L. Vinograd, B.V. Grinyov, V.I. Goriletsky, Effect of surface conditions on the light collection in scintillation detectors // Functional Materials, 4, №4, p.572-577, (1997). 